\title{
Optimization of electrical grid protection by a differential evolution algorithm
}

\author{
E. Zio \\ Ecole Centrale Paris- Supelec, Paris, France \\ Politecnico di Milano, Milan, Italy
}

L. R. Golea, G. Sansavini

Politecnico di Milano, Milan, Italy

\begin{abstract}
A modified binary differential evolution scheme is devised to identify an optimal protection strategy that minimizes the consequences of cascading failures on an electrical grid. The $380 \mathrm{kV}$ Italian power transmission network is employed as a realistic test case for the suggested protection strategy.
\end{abstract}

\section{INTRODUCTION}

We consider network protection (NP) by line switching by the system operator, following an attack or a failure to given components. Line switching is common practice for handling line overloads (Granelli et al. 2006), avoiding loss and/or cost reductions (Schnyder \& Glavitsch 1990), improving system security (Schnyder \& Glavitsch 1988), and the combination of these (Shao \& Vittal 2005). NP aims at hindering failure propagation by cutting off the possible "directions" along which failures can spread within the network. The strategy for such protection stands on the possibility of finding the optimal set of lines to be switched-off in order to limit the consequences of cascade failures; in large networks, this poses a combinatorial optimization problem.

In this paper, we address the NP optimization problem by a modified version of the binary differential evolution (MBDE) algorithm (Wang et al. 2010). Differential Evolution (DE) is an emerging population-based stochastic optimization technique (Storn \& Price 1995). The standard DE algorithm is simple and efficient and has been successfully applied in various scientific and engineering fields (Greem \& Kim 2001, Mahdavi et al. 2007, Coelho \& Bernert 2008, Saka 2009), often with superior performance than alternative optimization algorithms, e.g. particle swarm optimization and GAs (Ponsich \& Coello 2009). MBDE is a novel binary version of DE developed to tackle binary-coded optimization problems (Wang et al. 2010).

The goodness of the line switching solutions proposed during the MBDE search is evaluated by a model of cascading failure process which relies on a topological representation of the network connection pattern (Zio \& Sansavini 2009). This modeling en- tails considering the interplay between structural characteristics and dynamical aspects and provides indications on the elements critical for the propagation process (Zio \& Sansavini 2010), and on the actions that prevent or mitigate undesired effects (Motter 2004). Moreover, these models have shed light on the way complex networks react to faults and attacks, evaluating their consequences when propagation dynamics are taken into account. The system response often results in a dramatic cascade phenomenon due to avalanches of node outages.

The rest of the paper is organized as follows. Section 2 describes the basis of the model of cascading failures. The description of the MBDE algorithm is provided in Section 3. Section 4 presents the suggested NP strategy. The results of the NP optimization framework applied to a realistic case study, i.e. the Italian $380 \mathrm{kV}$ power transmission grid, are summarized in Section 5. Section 6 provides conclusions and points out directions for future work.

\section{THE MODEL OF CASCADING FAILURES IN NETWORK SYSTEMS}

We adopt a topological representation of the power grid as a network of $N$ nodes (substations or buses) and $K$ edges (transmission lines). Two types of buses are distinguished here: $N_{G}$ generators, i.e. sources for power, and $N_{D}$ distribution nodes (loads or primary substations), i.e. at the outer edge of the transmission grid. In order to abstractly account for the flow of the physical quantities in the network, we assume that the power is transmitted along the generatordistributor shortest paths (Batagelj 1994, Motter \& Lai 2002, Albert et al. 2004, Newman \& Girvan 2004, Latora \& Marchiori 2005, Duenas-Osorio \& Vemuru 2009, Zio \& Sansavini 2010). 
We model the load on a component, e.g. a bus or transmission line, as dependent on the number of shortest paths transiting through it, when flow is sent from each available generation node to each distribution node. In this view, the load, $L_{j}$, or amount of flow passing through a node, is measured by the node betweenness (Sabidussi 1966, Nieminen 1974, Freeman 1978, Freeman et al. 1991, Little 2002), calculated as the number of generator-distributor shortest paths that pass through that node. Assuming that power is routed through the most direct path, the betweenness of a bus gives an approximation of the amount of power flowing through it (Albert et al. 2004). Likewise, the load, $L_{i j}$, or amount of flow passing through an edge, is measured by the edge betweenness (Girvan \& Newman 2002). Node/edge betweenness measures the influence of a node/edge with respect to the flow of information in the network, especially in cases where the flow of information follows the available generator-distributor shortest path (Albert et al. 2004).

In order to assess the status of a component, its load is compared with the component capacity, i.e. the maximum load that it can process. The capacity is limited by technological constraints and economic factors. The capacity of node $j$ is dimensioned proportionally to its nominal load $L_{j}$ at which it is designed to operate, $C_{j}=(1+\alpha) L_{j}$. Likewise, the capacity of link $i j$ is dimensioned proportionally to its nominal load $L_{i j}$ at which it is designed to operate, $C_{i j}=(1+\alpha) L_{i j}$, where $\alpha>0$ is the tolerance parameter of the power transmission network. This parameter can be regarded as an operating margin allowing safe operations of the component under possible load increments. When $\alpha=0$, the system is working at its limit capacity, its operating margin being null: any further load added to a component would result in its failure and, possibly, in the propagation of a cascading failure involving a large part of the system.

When a component fails, it ceases to provide its service and is removed from the system. This leads to a redistribution of the shortest paths in the network and, consequently, to a change in the loads of the components remained active. If upon redistribution of the shortest paths, the load on a component increases beyond its capacity, the component fails and a new redistribution follows. The cascade process of successive failures is a function of the nodes states and also of the edge states, extending a common reference model (Batagelj 1994, Motter \& Lai 2002, Albert et al. 2004, Newman \& Girvan 2004, Latora \& Marchiori 2005, Duenas-Osorio \& Vemuru 2009, Zio \& Sansavini 2010).

In this work, the effects of cascading failures are summarized by two indicators, the connectivity loss, $C_{L}$, and the cascade size, $S$. The former quantifies the decrease of the ability of distribution substations to receive power from generators; its calculation re- lies on the topological structure of the network and the available "least-resistance" pathways:

$C_{L}=1-\frac{1}{N_{D}} \sum_{i=1}^{N_{D}} \frac{N_{G}^{i}}{N_{G}}$

where $N_{G}$ and $N_{D}$ represent the numbers of generator and distribution nodes in the unperturbed state of the network, and $N_{G}^{i}$ represents the number of generation units able to supply flow to distribution node $i$ after failures take place.

While the failure of lines only contributes to the further propagation of the cascade through load redirection, the failure of buses implies also the inability to provide electrical load to areas of the network. For this reason, we select to measure the disruption caused by the failure cascade by the number of nodes made inactive by the cascade, through $S$, and by the inability of generator nodes to power distributor nodes, through $C_{L}$.

\section{MODIFIED BINARY DIFFERENTIAL EVOLUTION ALGORITHM}

An improved binary DE (Wang et al. 2010) is used for optimizing the NP strategy.

DE has been originally developed as a populationbased global optimization algorithm for real-valued optimization problems (Storn and Price 1995). The original idea of DE lies in the way of perturbing the current population: this is obtained by adding to a chromosome the weighted difference between two others randomly selected from the population. The initial population is randomly generated by sampling values uniformly distributed between the lower and upper bounds of each of the decision variables. Three evolutionary operations, i.e., mutation, crossover and selection are used to update the population. During the first operation, the mutated individual $v_{i}$, called noisy vector, is generated according to the following equation

$v_{i}^{t+1}=x_{r_{1}}^{t}+F \cdot\left(x_{r_{2}}^{t}-x_{r_{3}}^{t}\right)$

where $F$, a positive real constant, is the scaling factor, $t$ is the index of generation and $x_{r_{1}}^{t}, x_{r_{2}}^{t}$ and $x_{r_{3}}^{t}$ are three randomly chosen individuals, i.e. target vectors, with indexes $r_{1} \neq r_{2} \neq r_{3} \neq i$.

After mutation, the noisy vector is further modified by the crossover process, in which the noisy and target vectors are mixed according to some rule to create the trial vector $u_{i}$. The trial vector inherits from the noisy and the target vectors different pieces of chromosome, as regulated by the crossover rate $C R$. The commonly used binomial crossover is defined as: 
$u_{j i}^{t+1}=\left\{\begin{array}{l}v_{j i}^{t+1}, \text { if } U(0,1] \leq C R \text { or } j=\operatorname{irand}(P) \\ x_{j i}^{t}, \text { if } U(0,1]>C R \text { and } j \neq \operatorname{irand}(P)\end{array}\right.$

$\forall j \in\{1,2, \ldots, K\}$

where $U(0,1]$ is a uniform continuous random value $\epsilon[0,1]$, $\operatorname{irand}(P)$ is a uniform discrete random number in the set $\epsilon\{1,2, \ldots, P\}, P$ is the population size, $j$ is the index of the dimensionality and $K$ is the length of the chromosome.

The selection operator is defined as:

$$
\begin{aligned}
& x_{i}^{t+1}=\left\{\begin{array}{l}
u_{i}^{t+1}, \text { if } \mathrm{f}\left(u_{i}^{t+1}\right)<\mathrm{f}\left(x_{i}^{t}\right) \\
x_{i}^{t}, \text { otherwise }
\end{array}\right. \\
& \forall i \in\{1,2, \ldots, P\}
\end{aligned}
$$

Referring to a single objective (SO) minimization, if the fitness value of the trial individual is better than the target one, it replaces the target individual; otherwise, the target individual is preserved in the next generation. This criterion ensures that the next generation is better than or at least equal to the previous one.

In the NP optimization problem here of interest, the decision variable vector $x$ is composed of $K$ elements corresponding to the network links; each element contains 1 if the corresponding line is to be switched-off, 0 otherwise. The objective function of the optimization is $C_{L}(x)$.

We define the attack/failure scenario whose consequences have to be minimized via an optimal NP strategy. We consider the attack to/failure of a single component whose following cascading process maximizes the damage to the network.

\section{NETWORK PROTECTION STRATEGY}

In practice, we identify the component whose removal yields the largest disruption in the network. $\mathrm{NP}$ aims at minimizing the effects of the cascade failure propagation by switching off lines after the cascade is triggered. Within the modeling framework here adopted, the cascade unfolds rapidly, through the shortest paths.

The NP strategy consists in taking action only once after the cascade is triggered, by opening several lines to prevent failure propagation. The issue is to decide which set of lines to disconnect in order to protect most from the cascade propagation.

The protection optimization seeks interventions that minimize the objective function $C_{L}(x)$, i.e. the connectivity loss of the network configuration $x=\left\{x_{j} \mid x_{j}\right.$ $\epsilon\{0,1\}, j=1, \ldots, K\}$, where $x_{j}=1$ if link $j$ is operating, or 0 otherwise.
Initially, all components, $N$ nodes and $K$ links, are characterized by initial loads $L_{i}$ and $L_{i j}$, respectively, and maximum capacities, $C_{i}$ and $C_{i j}$, respectively. The optimization is carried out by the MBDE algorithm, that uses binary variables to characterize the line switching decisions, as detailed in Section 3.

The algorithm for simulating the combined effects of the cascading failures and protection strategy proceeds in successive propagation steps as follows.

0 . The initiating event occurs, i.e. the failure of the most critical component, which is removed from the network. Each component that is operating is tested for failure: for $i=1, \ldots, N$, if node $i$ is working and $L_{i}>C_{i}$ then node $i$ fails; if link $i j$ is working and $L_{i j}>C_{i j}$ then link $i j$ fails.

1. The operator intervenes by switching off a set of lines, in order to limit the cascade failure effects, expressed by the objective function. The MBDE optimization algorithm returns the most appropriate network configuration that minimizes the objective function at the end of the current step $t=1$.

2 . The components loads are re-distributed taking into account the new, protected network topology. Each component that is operating is tested for failure: for $i=1, \ldots, N$, if node $i$ is working and $L_{i}>$ $C_{i}$ then node $i$ fails; if link $i j$ is working and $L_{i j}>C_{i j}$ then link $i j$ fails.

3.The stage counter $t$ is incremented by 1 and the algorithm is returned to step 2 .

4.The algorithm stops when no more working nodes fail.

\section{RESULTS AND DISCUSSION}

The NP optimization framework of Sections 3 and 4 has been applied to the topological network of the $380 \mathrm{kV}$ Italian power transmission network (Figure 1). The $380 \mathrm{kV}$ Italian power transmission network is a branch of a high voltage level transmission, which can be modeled as a network of $N=127$ nodes $\left(N_{G}=30\right.$ generators and $N_{D}=97$ distributors) and $K=171$ edges.

Figures 2 and 3 show the load distribution on each component when the network operates at nominal conditions. Node 88 and link 104 (the line connecting nodes 75 and 88) handle the largest load in the system, i.e. the largest number of generatordistribution shortest paths under our modeling scheme. 


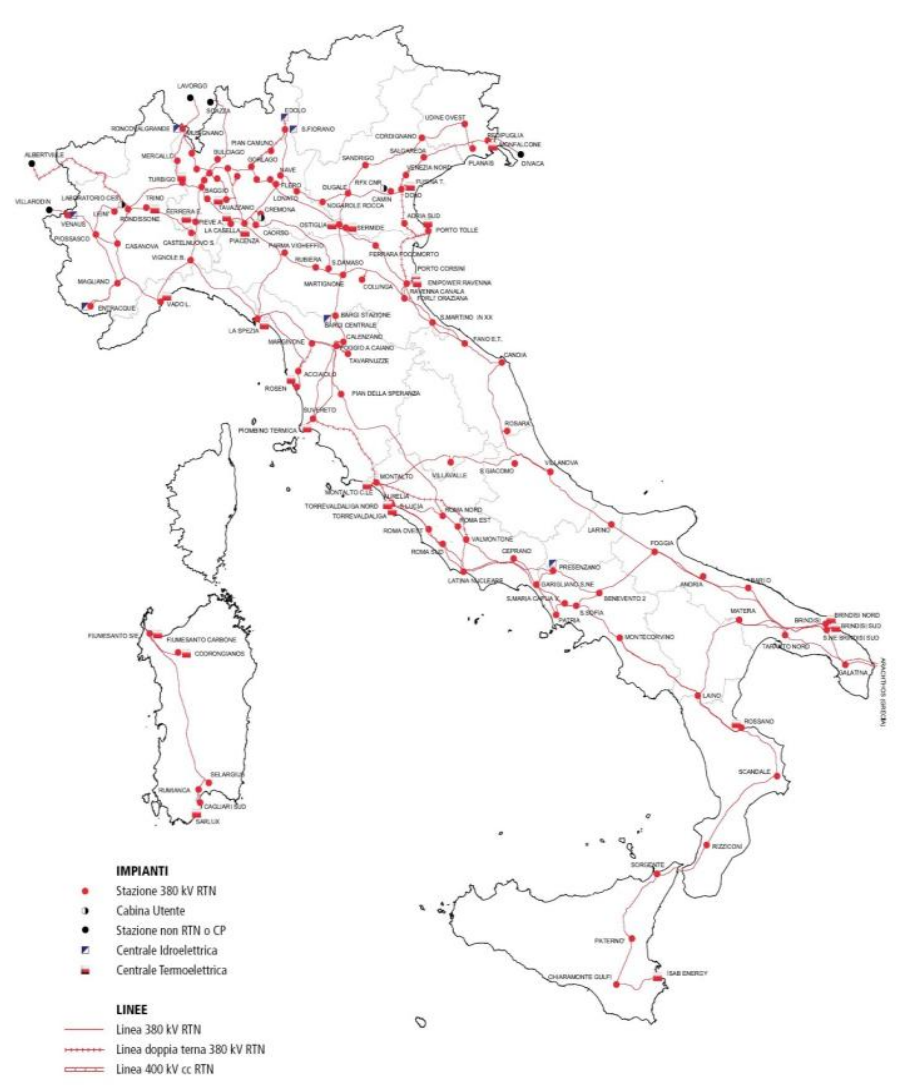

Figure 1. The $380 \mathrm{kV}$ Italian power transmission network (TERNA 2002, Rosato et al. 2007)

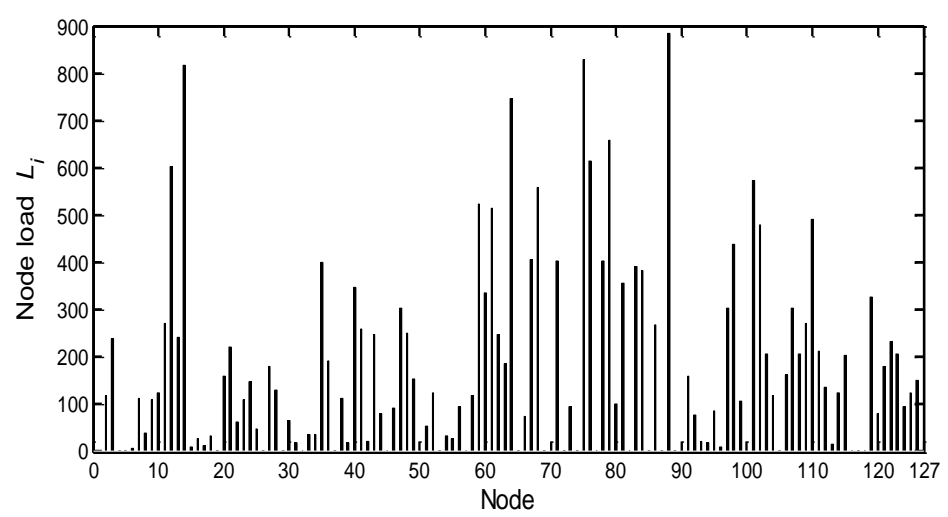

Figure 2. Loads on each node in the unperturbed configuration

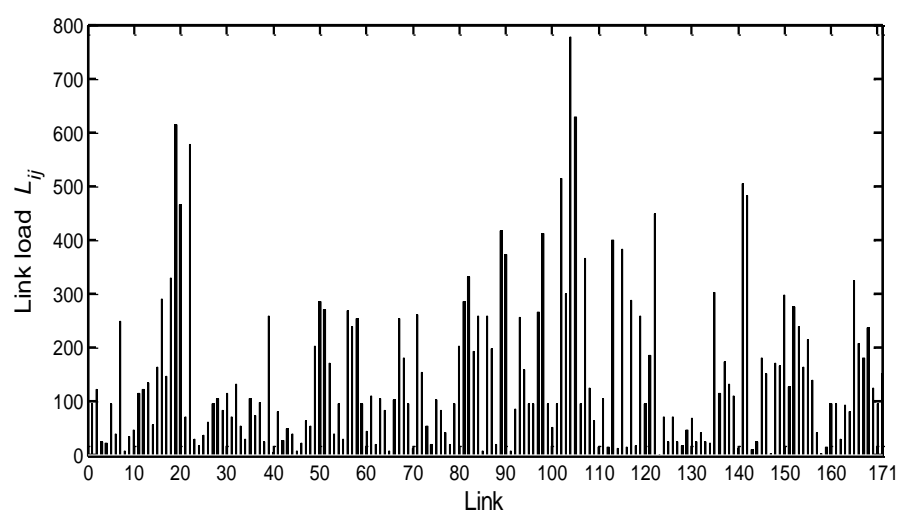

Figure 3. Loads on each line in the unperturbed configuration

The initiating event is identified as described in Section 4. Contrary to what can be supposed, it is found that the largest damages to the network are due to removals of components that are not among the most loaded (Figures 4-7). The removals of nodes 86 and 78 have the largest impacts on the network with respect to both $C_{L}$ and $S$. The failure of the most loaded node, i.e. node 88 , leads to cascades involving 28 components, compared to 48 components that fail when node 86 triggers the cascade. Also, $C_{L}=0.77$ when the system stabilizes if node 88 is removed, but $C_{L}=0.94$ when node 78 fails. The criticality of nodes 86 and 78 with respect to cascading failures is due to their position in the network, in proximity of highly loaded nodes.

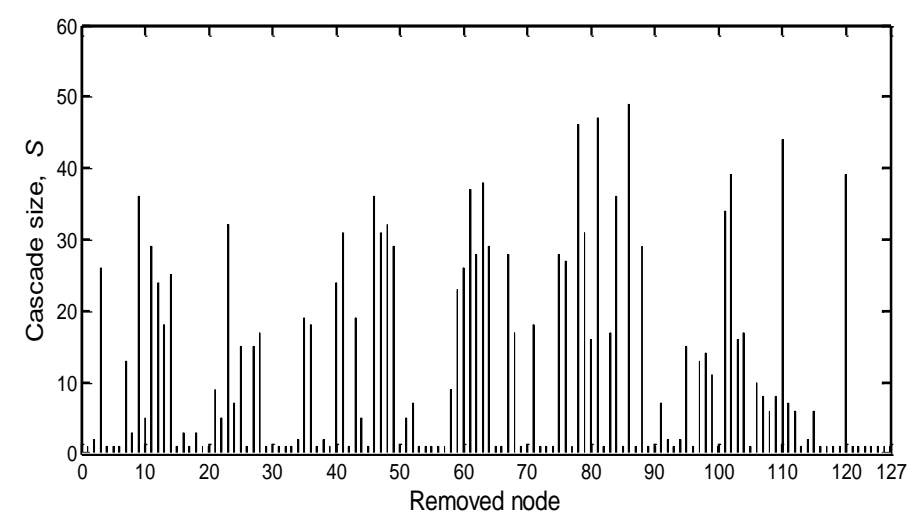

Figure 4 . The cascade size, $S$, caused by the removal of each node (abscissa) in the power transmission system of Figure 1

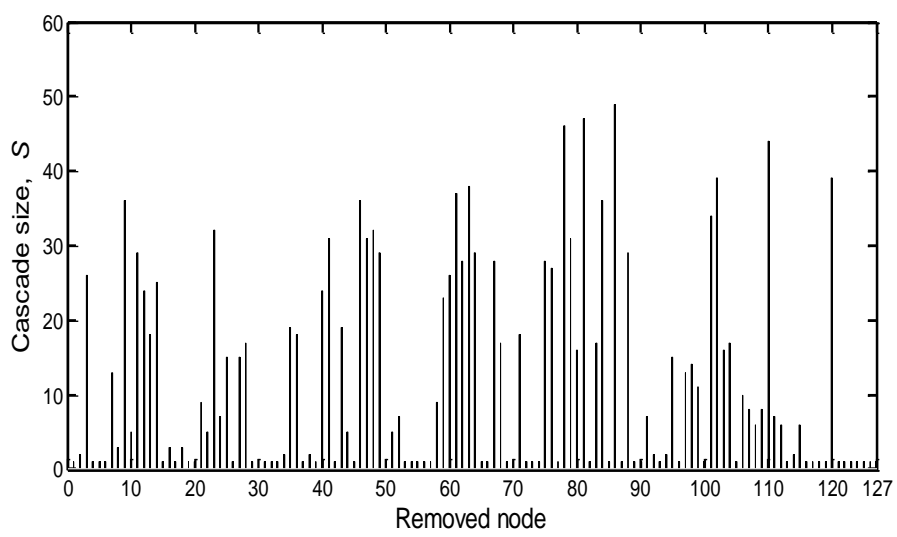

Figure 5. The connectivity loss, $C_{L}$, caused by the removal of each node (abscissa) in the power transmission system of Figure 1

The effects of cascading failures are investigated also with respect to the loss of lines. The removal of link 107 connecting nodes 78 and 81, results in the largest damage in terms of $S$ (57 nodes fail at the end of the cascade failure propagation) and $C_{L}=0.96$, while the failure of the most loaded link, i.e. 104, leads to $S=27$ components and $C_{L}=0.71$. The average initial load has little influence on the criticality, whereas the neighbors load and the failure of the connection between node 78 and 81 , have a large impact (node 78 is found to be a critical node in triggering the cascading failure propagation). 


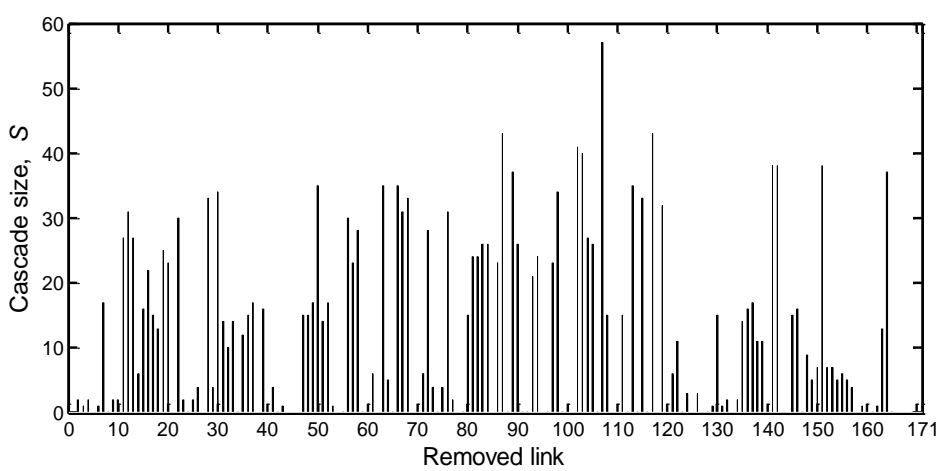

Figure 6 . The cascade size, $S$, caused by the removal of each link (abscissa) in the power transmission system of Figure 1

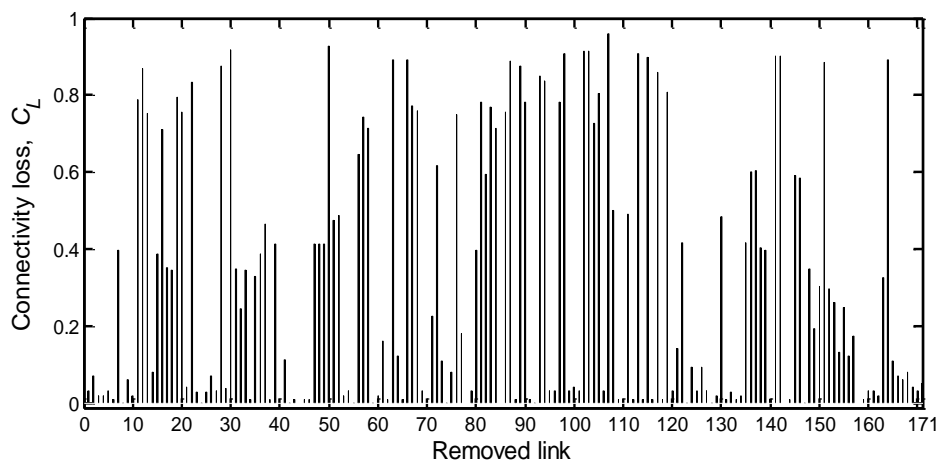

Figure 7 . The connectivity loss, $C_{L}$, caused by the removal of each link (abscissa) in the power transmission system of Figure 1

We observe that, on average, the loss of links results in a more severe damage than that caused by the loss of nodes, both in terms of $C_{L}$ and $S$. This is due to the fact that failure of a link causes the redistribution of the components loads to other working components (nodes and links). The disconnection of the transmission line (edge) 107 is identified as the worst-case scenario.

\subsection{Protection}

The cascade propagation is triggered at $t=0$ by an attack/failure consisting in the disruption of link 107, and produces the loss of six nodes by the end of the first propagation step, $t=1$. We aim at reducing the effects of the continuation of the cascade of failures in the network through the application of the protection strategy. The optimization parameters are summarized in Table 1.

Table 1. The parameters of the MBDE algorithm

\begin{tabular}{ll}
\hline \multicolumn{2}{c}{ MBDE parameters } \\
\hline Population size $P$ & 40 \\
Dimensionality of solutions $K$ & 171 \\
Crossover rate $C R$ & 0.8 \\
Scaling factor $F$ & 0.2 \\
Minimum fitness error & $10^{-4}$ \\
Maximum number of generation & 1500 \\
\hline
\end{tabular}

The optimal NP strategy requires disabling 27 lines, i.e. $21 \%$ of the network connections (Table 2, column 2). This represents a feasible number of lines to be switched-off and it is consistent with the size of other protection strategies reported in the literature. As an example, (Salmeron et al. 2004) suggests a protection strategy that involves the hardening of $50 \%$ of lines in a network characterized by 24 nodes and 40 lines.

The optimal set of lines identified in the protection strategy is represented in Figure 8. The lines are spread over the entire network with a major concentration in the northern part. By adopting this protection strategy, the operator intervention maintains a good level of working components with acceptable $C_{L}$.

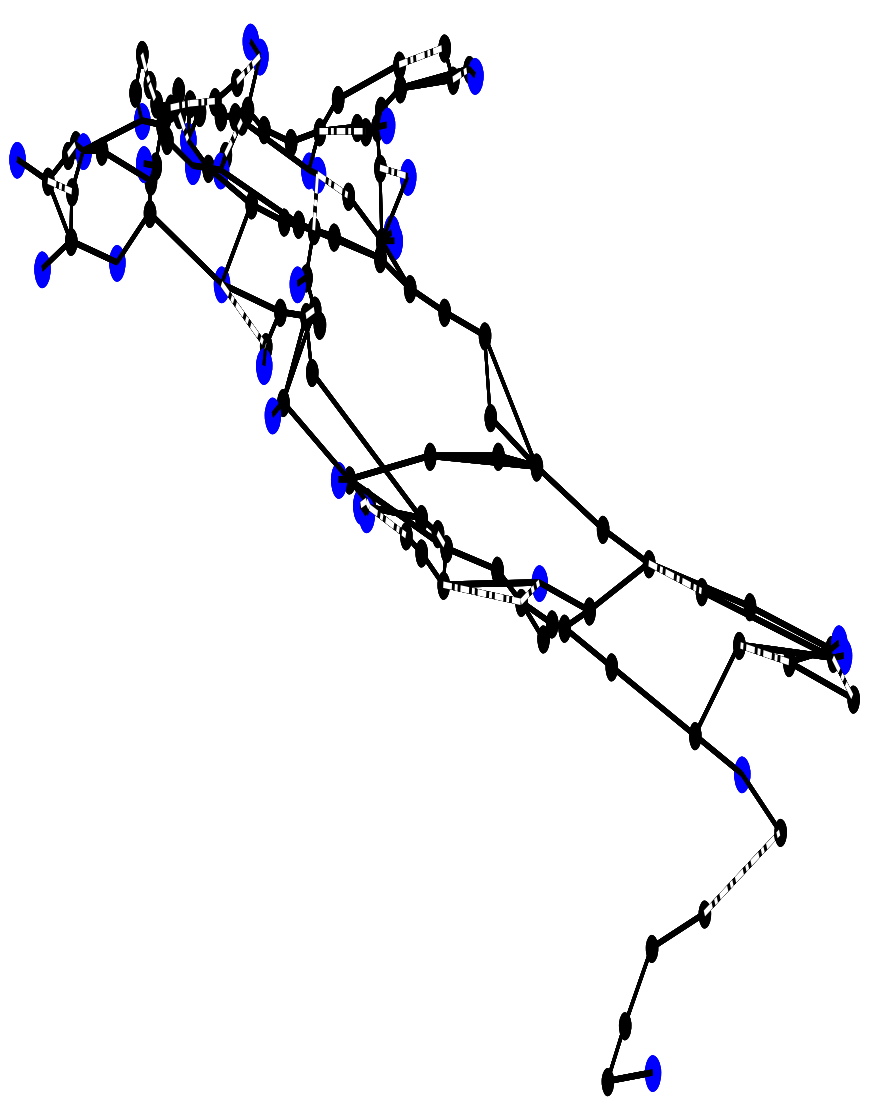

Figure 8 . The dotted lines in this graphic representation of the network are the optimal set of 27 edges to be switched-off to optimally contain the failure cascade

Figure 9 presents the effects of the cascade after the initial failure, without any operator intervention (dotted line) and with the operator intervention of switching off the optimal set of 27 lines, both in terms of $S$ (left graph) and $C_{L}$ (right graph).

The effects of the NP intervention can be evinced by comparing the evolutions in Figure 9. The optimal line switching intervention at $t=1$ reduces $S$ and $C_{L}$ by $87.7 \%$ and by $34.6 \%$, respectively. The failures keep propagating in the network and the cascade ends at $t=3$. The results obtained when the system 
is stabilized are satisfactory: $S$ is reduced from 57 nodes to 10 nodes, while $C_{L}$ is improved by approximately $21 \%$. There are four nodes that fail after the operator intervenes with the protection strategy, i.e. Pieve A substation, situated in the Lombardy region and Rondissone, Trino and Turbigo substations, situated in the Piemonte region (Figure 1).
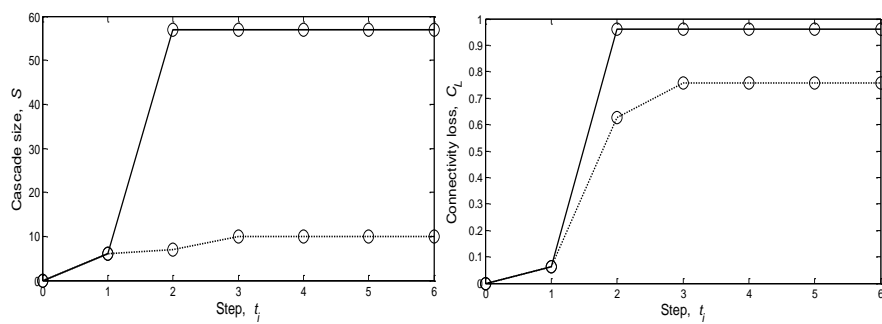

Figure 9. The cascade evolution in terms of cascade size, $S$, and connectivity loss, $C_{L}$, after the failure of line 107 and with no intervention at $t=1$ (solid lines) or optimal operator intervention at $t=1$ (dotted lines)

The results obtained at the end of the optimization are summarized in Table 2.

Table 2. Best optimal switching scheme

\begin{tabular}{|c|c|c|c|}
\hline \multirow{2}{*}{$\begin{array}{l}\text { Number } \\
\text { of lines }\end{array}$} & \multirow{2}{*}{$\begin{array}{l}\text { Optimal line switching } \\
\text { scheme }\end{array}$} & \multicolumn{2}{|c|}{ Connectivity loss, $C_{L}$} \\
\hline & & $\begin{array}{l}\text { computational } \\
\text { time } t=1\end{array}$ & $\begin{array}{l}\text { end of } \\
\text { cascade }\end{array}$ \\
\hline 27 & 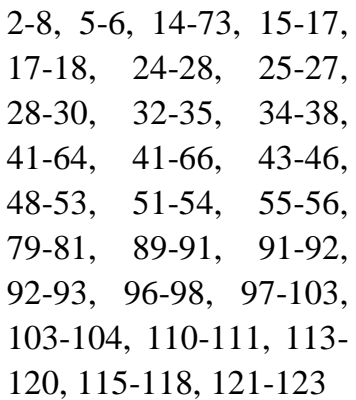 & 0.608 & 0.758 \\
\hline
\end{tabular}

\section{CONCLUSIONS}

We have considered the optimal protection of power networks by extending a previously developed model for cascading failures propagation and embedding it in a MBDE scheme of optimization. The worstcase initiating failure scenario is determined by looking at the effects of the cascading failures triggered by the removal of single nodes or links. The optimal protection strategy of line switching is identified by MBDE so as to limit the cascade failure consequences.

Future research steps will extend the abstract model of cascading failures beyond network abstraction to incorporate physical properties and time dynamics of power transmission. Within this framework, other interdiction scenarios and multiple protection strategies can be developed. In particular, an iterative NP strategy can be implemented, that incorporates realtime constraints arising from the network and load configuration updates given previous protective actions.

\section{ACKNOWLEDGEMENTS}

This work was funded by the Foundation pour une Culture de Securite Industrielle of Toulouse, France, under the research contract $\mathrm{AO} 2006-01$.

\section{REFERENCES}

Albert, R., Albert, I. \& Nakarado, G.L. 2004. Structural vulnerability of the North American power grid, Physical Review E 69, 025103(R).

Batagelj, V. 1994. Semirings for social networks analysis, Journal of Mathematical Sociology 19(1): 53-68.

Babu, B.V., Gujarathi, A.M., Katla, P. \& Laxmi, V.B. 2007. Strategies of Multi-Objective Differential Evolution (MODE) for Optimization of Adiabatic Styrene Reactor. In Proceedings of the International Conference on Emerging Mechanical Technology: Macro to Nano (EMTMN-2007); p 243.

Coelho, L.S. \& Bernert, D.L.A. 2008 An improved harmony search algorithm for synchronization of discrete-time chaotic systems, Chaos Solitons Fract.

Dueñas-Osorio, L. \& Vemuru, S.M., Cascading failures in complex infrastructures systems. Structural safety 31: 157167.

Freeman, L.C., Centrality in social networks conceptual clarification. Social Networks 1(3): 215-239.

Freeman, L.C., Borgatti, S.P. \& White, D.R., Centrality in valued graphs: A measure of betweenness based on network flow, Social Networks 13(2): 141-154,1991.

Girvan, M. \& Newman, M.E.J., Community structure in social and biological networks, Proc. Natl. Acad. Sci., USA 99, 5890, 2002.

Granelli, G., Montagna, M., Zanellini, F., Bresesti, P., Vailati, R., \& Innorta, M., Optimal network reconfiguration for congestion management by deterministic and genetic algorithms, Elect. Power Syst. Res., vol. 76, pp. 549-556, Apr. 2006.

Geem, Z.W., Kim, J.H. \& Loganathan, G.V., A new heuristic optimization algorithm: harmony search, Simulation 2001; $76(2): 60-8$.

Latora, V. \& Marchoiri, M., Vulnerability and protection of infrastructure networks. Physical Review E 71, 015103.

Little, R.G., Controlling Cascading Failure: Understanding the Vulnerabilities of Interconnected Infrastructures. Journal of Urban Technology 9(1): 109 - 123, 2002.

Mahdavi, M., Fesanghary, M. \& Damangir, E., An improved harmony search algorithm for solving optimization problems, Appl Math Comput 2007; 188(2):1567-79.

Motter, A.E. \& Lai, Y.-C., Cascade-based attacks on complex networks. Physical Review E 66(6): 065102, 2002.

Motter, A.E., Cascade control and defense in complex Networks, Physical Review Letters, vol. 93, $\mathrm{nr}$ 9, pp. 098701(1-4), 2004.

Newman, M.E.J. \& Girvan, M., Finding and evaluating community structure in networks. Physical Review E 69(2): 026113, 2004.

Nieminen, J., On the centrality in a graph. Scandinavian Journal of Psychology 15(1): 332-336, 1974. 
Ponsich, A. \& Coello, C.A., Differential Evolution performances for the solution of mixed integer constrained Process Engineering problems, Applied Soft Computing (2009), doi:10.1016/j.asoc.2009.11.030.

Sabidussi, G., The centrality index of graphs, Psychometrika, 31(4), pp. 581-603, 1966.

Saka, M.P., Optimum design of steel sway frames to BS5950 using harmony search algorithm, J Constr Steel Res 2009; 65(1):36-43.

Salmeron, J., Wood, K. \& Baldick, R., Analysis of Electric Grid Security Under Terrorist Threat, IEEE Transactions on power systems, Vol. 19, no. 2, May 2004.

Schnyder, G. \& Glavitsch, H., Integrated security control using an optimal power flow and switching concepts, IEEE Trans. Power Syst., vol. 3, no. 2, pp. 782-790, May 1988.

Schnyder, G. \& Glavitsch, H., Security enhancement using an optimal switching power flow, IEEE Trans. Power Syst., vol. 5, no. 2, pp. 674-681, May 1990.

Shao, W. \& Vittal, V., Corrective switching algorithm for relieving overloads and voltage violations, IEEE Trans. Power Syst., vol. 20, no. 4, pp. 1877-1885, Nov. 2005.

Storn, R. \& Price, K., Differential evolution - A simple and efficient adaptive scheme for global optimization over continuous spaces, Technical Report TR-95-012, International Computer Science Institute, Berkeley, CA. 1995.

Talukdar, S.N., Apt, J., Ilic, M., Lave, L.B. \& Morgan, M.G:, Cascading Failures: Survival versus Prevention, The Electricity Journal, Vol. 16, Issue 9, November 2003, pp. 25-31.

Wang, L., Fu, X., Menhas, M.I. \& Fei, M., A Modified Binary Differential Evolution Algorithm, Life System Modeling and Intelligent Computing, Lecture Notes in Computer Science, 2010, Volume 6329/2010, 49-57, DOI: 10.1007/9783-642-15597-0_6.

Zio, E. \& Sansavini, G., Modeling failure cascade in network systems due to distributed random disturbances. Proceedings ESREL 2008. Martorell et al. (eds): Safety, Reliability and Risk Analysis: Theory, Methods and Applications. CRC Press, Taylor \& Francis Group, London, 2009.

Zio, E. \& Sansavini, G., Modeling failure cascades in critical infrastructures with physically-characterized components and interdependencies, in Reliability, Risk and Safety: Back to the Future - Ale, Papazoglou \& Zio (eds), pp. 652 - 661, Proceedings of ESREL 2010 Europe Annual Conference, 59 September 2010, Rhodes, Greece, Taylor \& Francis Group, London, 2010.

Zio, E. \& Viadana, G., A thorough analysis of Differential Evolution in single-objective and multi-objective optimization, submitted to The Seventh International Conference on" Mathematical Methods in Reliability"- Theory. Methods. Applications, June 20-24, 2011. 